\title{
Novel nomogram-based integrated gonadotropin therapy individualization in in vitro fertilization/ intracytoplasmic sperm injection: A modeling approach
}

\author{
Abdel Hameed I M Ebid' ', Sara M Abdel Motaleb', Mahmoud I Mostafa', Mahmoud M A Soliman² \\ 'Department of Pharmacy Practice, Helwan University, Cairo; ${ }^{2}$ Department of Obstetrics and Gynecology, Cairo University, Cairo, Egypt
}

\begin{abstract}
Objective: This study aimed to characterize a validated model for predicting oocyte retrieval in controlled ovarian stimulation (COS) and to construct model-based nomograms for assistance in clinical decision-making regarding the gonadotropin protocol and dose.

Methods: This observational, retrospective, cohort study included 636 women with primary unexplained infertility and a normal menstrual cycle who were attempting assisted reproductive therapy for the first time. The enrolled women were split into an index group ( $n=497)$ for model building and a validation group $(n=139)$. The primary outcome was absolute oocyte count. The dose-response relationship was tested using modified Poisson, negative binomial, hybrid Poisson- $\mathrm{E}_{\max }$ and linear models. The validation group was similarly analyzed, and its results were compared to that of the index group.

Results: The Poisson model with the log-link function demonstrated superior predictive performance and precision (Akaike information criterion, 2,$704 ; \lambda=8.27$; relative standard error $(\lambda)=2.02 \%)$. The covariate analysis included women's age $(p<0.001)$, antral follicle count $(p<0.001)$, basal follicle-stimulating hormone level $(p<0.001)$, gonadotropin dose $(p=0.042)$, and protocol type $(p=0.002$ and $p<0.001$ for short and antagonist protocols, respectively). The estimates from 500 bootstrap samples were close to those of the original model. The validation group showed model assessment metrics comparable to the index model. Based on the fitted model, a static nomogram was built to improve visualization. In addition, a dynamic electronic tool was created for convenience of use.

Conclusion: Based on our validated model, nomograms were constructed to help clinicians individualize the stimulation protocol and gonadotropin doses in COS cycles.
\end{abstract}

Keywords: Dose-response relationship; Oocytes; Ovarian stimulation; Patient-specific modeling

\section{Introduction}

Improvements in procedural and therapeutic strategies in assisted reproductive technology (ART) have led to a dramatic increase in live birth rates and reduced rates of cycle cancellation [1]. However, poor

Received: June 23, 2020 - Revised: December 8, 2020 • Accepted: December 10, 2020 Corresponding author: Mahmoud I Mostafa

Department of Pharmacy Practice, Helwan University, Wadi Hof, Cairo 11795, Egypt Tel: +2-1006605563 Fax: +2-25541845

E-mail:mahmoud.ibrahim@pharm.helwan.edu.eg

This is an Open Access article distributed under the terms of the Creative Commons Attribution Non-Commercial License (http://creativecommons.org/licenses/by-nc/4.0/) which permits unrestricted non-commercial use, distribution, and reproduction in any medium, provided the original work is properly cited. ovarian response or hyper-response remain limitations. Advances in the design of stimulation protocols with milder properties have reduced the costs of treatment, enhanced oocyte retrieval [2], and led to a significant reduction of the hyper-response rate [3]. Nevertheless, these protocols have been criticized for their possible reduced efficacy in terms of reduced pregnancy and live birth rates [4]. The trade-off between controlled ovarian stimulation (COS) safety and efficacy is further complicated by the complex exposure-response relationship between ovarian response and the gonadotropin dose in each protocol [5]. Therefore, the difficulty in the choosing a stimulation protocol and subsequent gonadotropin dose is a major challenge in designing ART cycles, and it is necessary to personalize COS 
based on the patient's specific factors to optimize both the safety [6] and efficacy [7] of the stimulation. Currently published models for personalized gonadotropin therapy have identified women's age, gonadotropin dosage, and ovarian reserve tests (ORTs), including anti-Müllerian hormone (AMH) levels, follicle-stimulating hormone (FSH) levels, and antral follicular count (AFC), as the most significant predictors of ovarian response [8-11]. Nevertheless, these models have many drawbacks, limiting their validity for application in clinical practice.

First, most of these models define ovarian response as high, good, or poor based on logistic regression models. Different thresholds of ovarian response for each category may potentially bias the predicted response, resulting in misclassification errors [12]. Moreover, modeling the response using categorical variables hides the extensive patterns of variability embedded within each category, resulting in potential information loss [13]. Second, the gonadotropin doseoocyte relationships have been described using different structural models, with no consensus on which one is optimal. Third, the complex mathematical calculations involved in the final model equations limit the practical application of these models in real-world settings. Fourth, models predicting the probability of COS success in terms of pregnancy rates or live birth rates are potentially confounded by the laboratory phase and luteal phase of embryo transfer, regardless of the specific treatment or clinical factors. Therefore, the current study aimed to test different structural models of the gonadotropin dose-oocyte relationship, to address the limitations of the current models, and to translate the resultant model into an easy-to-use and clinically applicable tool.

\section{Methods}

\section{Study design}

This was an observational, single-center, retrospective cohort study. The study group was split into an index cohort, which was analyzed for model building and optimization, and a validation cohort, which was used for model validation. The candidate women were randomly allocated to these two groups in a 4 to 1 ratio $(80 \%$ index, $20 \%$ validation). Any additional patients included in the analysis phase were assigned to the validation group. The study followed the precepts of the Helsinki Declaration, and was approved by the Ethical Committee of the Faculty of Pharmacy, Helwan University. Written informed consent was obtained from the recruited women.

\section{Patients}

Women undergoing intracytoplasmic sperm injection (ICSI) between January 2015 and May 2019 at Midwest Reproductive Center (Giza, Egypt) were assessed for eligibility to be included in the study.
The inclusion criteria were an adequate trial of spontaneous conception for at least 1 year, the first trial of in vitro fertilization (IVF)/ICSI cycles, and normal menstrual cycle. Patients were excluded if they were more than 40 years old or presented with a secondary cause of female infertility, in order to avoid the potential confounding of their underlying pathologies or special treatment plans with our model. Therefore, women who presented with any explained cause of infertility, including tubal, uterine, or ovarian pathologies, as well as those with neurological or endocrine causes of infertility, were excluded. Moreover, women were excluded if they reported recent administration of hormonal contraceptives within the last 3 months. Since conventional IVF was not available at our center, all candidate women underwent standard IVF/ICSI. Data retrieved from the local center registry included basal FSH measured on day 2-3 of the menstrual cycle, AMH levels, total AFC (including follicles measuring 2-10 mm), the starting and total gonadotropin dose, duration of the stimulation, type of the stimulation protocol, number of metaphase II oocytes, and the total count of oocytes retrieved following COS.

\section{Treatment protocols}

According to the treating physician's clinical experience, women underwent pituitary desensitization using a long, short, or antagonist protocol. For the long protocol, women were initiated on a gonadotropin-releasing hormone (GnRH) agonist (Triptorelin; Decapeptyl; Ferring Pharmaceuticals, Saint-Prex, Switzerland) on day 20 of the menstrual cycle preceding the stimulation cycle. When pituitary downregulation was attained, as evidenced by a serum estradiol level $<50 \mathrm{pg} / \mathrm{mL}$, gonadotropin stimulation was administered as human menopausal gonadotropin (Merional; IBSA, Lugano, Switzerland) or recombinant FSH (Gonal-F; Merck Serono, Eysins, Switzerland) started at empirical doses determined by the physician based on age, serum AMH levels, and body mass index (BMI) [14]. For the short protocol, a GnRH agonist was started on the first day of the ART cycle; then, gonadotropin doses were started on day 3 until the day of human chorionic gonadotropin (hCG) administration [15]. Finally, women on the antagonist protocol started gonadotropin stimulation doses daily on day 1 of the stimulation cycle (day 2-3 of the menstrual cycle). A GnRH antagonist (Cetrorelix; Cetrotide, Merck Serono) with a daily dose of $0.25 \mathrm{mg} /$ day (subcutaneous) was then initiated when at least 1 follicle measured $14 \mathrm{~mm}$ and continued until the target follicular response [4]. For each protocol, the starting gonadotropin dose ranged from 150 to $450 \mathrm{IU} /$ day and was modified subsequently if needed on a weekly basis in 75 IU increments according to the results of ultrasonographic follicular tracking. Final oocyte maturation was triggered with hCG (Choriomon, IBSA) administered at a dose of 10,000 $\mathrm{U}$ when at least three follicles measured $\geq 18 \mathrm{~mm}$, and ovum pick-up was then facilitated by transvaginal ul- 
trasonography 36 hours later. Embryo transfer was performed on days $2-4$ after pick-up.

\section{Ultrasonographic and hormonal measurements}

Basal antral follicles were measured in both ovaries by qualified radiographers on day 3 of the menstrual cycle using transvaginal ultrasonography (ClearVue 350; Philips, Louisiana, USA). Longitudinal AFC evaluation 1 week after the start of ovarian stimulation was carried out to guide the incremental gonadotropin dose modification. For hormonal assays, cubital vein blood samples were taken in the early follicular phase (day 3), prior to the start of ovarian stimulation regimens. Serum AMH was analyzed by enzyme-linked immunosorbent assay (ELISA) using the Beckman Coulter AMH ELISA kit (Immunotech, Marseilles, France). Levels of basal FSH were determined in a similar fashion using ELISA (Immulite 2000 analyzer; Siemens, Munich, Germany) on day 3 of the cycle. All samples were analyzed on the day of collection using the same kits and operators to minimize intra-assay variability.

\section{Outcomes}

The primary outcome investigated was the absolute count of oocytes retrieved following COS in ICSI cycles. The secondary outcomes were the rates of hypo- or hyper-response and the ovarian response prediction index (ORPI). Poor response was defined as the attainment of fewer than eight oocytes at the end of the stimulation cycle, whereas hyper-response was defined as the retrieval of 15 oocytes or more [6]. The ORPI was calculated as (AMH level $\times$ AFC)/age [9].

\section{Statistical analysis}

Continuous data are described as mean \pm standard deviation or median and interquartile range (IQR), while categorical variables are presented as the count with corresponding percentages $(n, \%)$. For comparisons, the Mann-Whitney U-test was used for continuous non-normally distributed data, the $t$-test for normally distributed data, and the chi-square test for ratios. An a priori alpha of less than 0.05 was set. The process of model development involved a threestep approach: structural model development, building a covariate model, and validation of the final model.

\section{1) Structural model development}

The hypothesized models for investigation of the dose-response relationship included modified Poisson, negative binomial, linear, and hybrid $\mathrm{E}_{\max }$ models with the log-link function for counts. The hybrid $\mathrm{E}_{\max }$ model is a proposed structural model that combines the $E_{\text {max }}$ model [16] and the Poisson model [17] in the same structure to account for non-linearity in the dose-response relationship between FSH doses and oocyte retrieval. The precision of the tested models was evaluated by comparing the estimated percentages of relative standard error (\%RSE) calculated for each model. To characterize the interindividual variability, mixed-effect modeling was applied at this stage.

\section{2) Covariate selection}

After the determination of the best structural model, potential covariates, including AFC, AMH and FSH levels, age, gonadotropin dose, and protocol type, were tested for their impact on the Akaike information criterion (AIC) of the structural model. Stepwise forward-inclusion covariate modeling was applied. The difference in AIC values between the candidate models was approximately chisquare-distributed and was considered significant when the AIC changed by more than 3.84 when testing a single covariate $\left(x^{2}\right.$, $\mathrm{df}=1 ; p=0.05)$.

\section{3) Model validation}

Non-parametric bootstrapping of the original dataset was performed with 500 replicates of the same sample size, and the results were compared to those of the base model. Furthermore, model metrics including the root mean squared error considering the log error model, C-index, chi-square model score, and pseudo- $R^{2}$ were calculated from the validation group analysis and compared to their corresponding estimates in the index group.

\section{Software}

Descriptive statistics and group comparisons were performed using IBM SPSS ver. 26.0 (IBM Corp., Armonk, NY, USA). The structural model assessment was performed using Monolix software ver. 2019R2 (Lixoft, Paris, France). Covariate modeling, validation, and nomogram development were performed using $R$ ver. 3.5.0 (R Foundation, Vienna, Austria).

\section{Results}

\section{Baseline and clinical characteristics}

In total, 636 women were enrolled in the present study, with a mean age of $29.87 \pm 5.26$ years. The median AFC was nine follicles (range, 1-33 follicles), while the mean AMH level was 2.54 \pm 1.31 $\mathrm{ng} / \mathrm{mL}$ (range, $0.6-14.9 \mathrm{ng} / \mathrm{mL}$ ), and the mean FSH level was $6.88 \pm 3.79 \mathrm{mlU} / \mathrm{mL}$ (range, 1.2-27.6 mlU/mL). COS was performed using long (32.9\%), short (56.6\%), or antagonist (10.5\%) protocols, resulting in poor (41.5\%), normal (50.6\%), and hyper-response (7.9\%). No patients presented with severe ovarian hyperstimulation syndrome. The starting gonadotropin dose applied in different COS cycles ranged between 150 and 450 IU. The majority of women received a dose of $225 \mathrm{IU}$ (54.6\%). The median number of retrieved oo- 
cytes at the end of stimulation was 8 (IQR, 6-11), and the mean units required to obtain one oocyte was $35.85 \pm 26.96 \mathrm{IU} /$ oocyte. Of the 636 patients, 497 (78\%) were assigned to the index group and 139 (22\%) to the validation group. Pairwise comparisons between the index group and the validation group demonstrated statistically non-significant differences. Table 1 summarizes the baseline characteristics of the study groups.

\section{Structural model building}

Among the screened dose-response models, the modified Poisson model (with log-dose) demonstrated the highest parameter precision $(\lambda=8.27, \operatorname{RSE}(\lambda)=2.02 \%)$, lowest unexplained interindividual parameter variability (28\%; RSE, $7.8 \%)$, and the best predictive performance as evidenced by the lowest AIC compared to all other models (AIC, 2,704). Our novel hybrid model demonstrated convincing precision to estimate the $\mathrm{E}_{\max }$ and $\mathrm{ED}_{50}$ parameters (\%RSE, $2.17 \%$ and $0.44 \%$, respectively); however, it was excluded due to an apparently higher AIC (AIC, 2,752). Finally, the linear models presented the worst performance, as shown by the highest AIC and \%RSE. A summary of the structural model assessment is provided in Table 2.

\section{Covariate selection}

Based on the modified Poisson structural model, univariate analysis was performed. Statistical significance was demonstrated for age, AFC, AMH and basal FSH levels, gonadotropin dose, and stimulation protocol type, while BMI was found to be a non-significant predictor $(p=0.57)$. Despite significance in the univariate analysis $(p<0.001)$, $\mathrm{AMH}$ was found to be nonsignificant in the multivariate model $(p=0.21)$, possibly due to multicollinearity with other covariates. Centering $\mathrm{AMH}$ around the median or correcting the independent variables for $\mathrm{AMH}$ resulted in imprecise estimates of model coefficients and poor model stability. Therefore, AMH was dropped from

Table 1. Baseline clinical and demographic characteristics of the study groups

\begin{tabular}{|c|c|c|c|c|}
\hline Baseline characteristics & Total $(n=636)$ & Index group $(n=497)$ & Validation group $(n=139)$ & $p$-value \\
\hline Age (yr) & $29.87 \pm 5.26$ & $29.97 \pm 5.27$ & $29.52 \pm 5.22$ & 0.38 \\
\hline Body mass index $\left(\mathrm{kg} / \mathrm{m}^{2}\right)$ & $29.58 \pm 3.41$ & $29.6 \pm 3.41$ & $29.47 \pm 3.44$ & 0.69 \\
\hline $\mathrm{AMH}(\mathrm{ng} / \mathrm{mL})$ & $2.54 \pm 1.31$ & $2.55 \pm 1.37$ & $2.49 \pm 1.08$ & 0.78 \\
\hline Basal FSH (mlU/mL) & $6.88 \pm 3.79$ & $6.89 \pm 3.85$ & $6.82 \pm 3.6$ & 0.82 \\
\hline ORPI & $0.65(0.35-1.24)$ & $0.64(0.34-1.25)$ & $0.68(0.37-1.2)$ & 0.71 \\
\hline Long & $209(32.9)$ & $166(33.4)$ & $43(30.9)$ & \\
\hline Short & $360(56.6)$ & $277(55.7)$ & $83(59.7)$ & \\
\hline Antagonist & $67(10.5)$ & $54(10.9)$ & $13(9.4)$ & \\
\hline Starting gonadotropin dose (IU) & & & & 0.22 \\
\hline 150 & $123(19.3)$ & $98(19.7)$ & $25(18)$ & \\
\hline 450 & $16(2.5)$ & $16(3.2)$ & 0 & \\
\hline Total gonadotropin dose (IU) & $2,844 \pm 1,710$ & $2,881 \pm 1,795$ & $2,710 \pm 1,361$ & 0.88 \\
\hline Duration of stimulation (day) & $11 \pm 3.34$ & $11 \pm 3.44$ & $10.8 \pm 2.93$ & 0.77 \\
\hline Gonadotropin dose adjustment & $121(19.2)$ & $96(19.3)$ & $25(18)$ & \\
\hline Dose step-up & $85(13.4)$ & $65(13.1)$ & $20(14.4)$ & 0.72 \\
\hline Dose step-down & $36(5.6)$ & $31(6.2)$ & $5(3.6)$ & 0.47 \\
\hline Starting gonadotropin units per oocyte & $35.85 \pm 26.96$ & $36.62 \pm 28.46$ & $33.08 \pm 20.59$ & 0.46 \\
\hline No. of retrieved oocytes & $8(6-11)$ & $8(6-11)$ & $9(6-11)$ & 0.21 \\
\hline No. of retrieved MII oocytes & $2(1-4)$ & $2(1-4)$ & $2(1-4)$ & 0.53 \\
\hline Response to ovarian stimulation & & & & 0.57 \\
\hline
\end{tabular}

Values are presented as mean \pm standard deviation, median (interquartile range), or number (\%).

$\mathrm{AFC}$, antral follicular count; AMH, anti-Müllerian hormone; FSH, follicle-stimulating hormone; ORPI, ovarian response prediction index; MII, metaphase II. 
the final multivariate model. The final multivariate regression model identified age, AFC, FSH levels, protocol type, and the starting gonadotropin dose as significant independent predictors of the oocyte retrieval count (Table 3). The gonadotropin dose initially demonstrated a negative relationship with oocyte count in the univariate analysis (regression coefficient, $-0.221 ; p<0.001$ ); however, after adjusting for confounding variables, a positive relationship was shown in multivariate regression (adjusted odds ratio, 1.11; 95\% confidence interval [CI], 1.004-1.24).

Table 2. Comparison of different structural models of the oocyte count (dependent variable) and gonadotropin dose (independent variable)

\begin{tabular}{|c|c|c|c|c|}
\hline Model & Parameter & Estimate & SE (\%RSE) & AIC \\
\hline \multirow[t]{2}{*}{ Modified Poisson } & $\lambda$ (oocyte) & 8.27 & $0.167(2.02)$ & 2,704 \\
\hline & $\operatorname{UIIV}(\lambda)$ & 0.28 & $0.022(7.8)$ & \\
\hline \multirow[t]{4}{*}{ Negative binomial } & $\lambda$ (oocyte) & 8.35 & $0.164(1.96)$ & 2,709 \\
\hline & o (oocyte) & 0.021 & $0.008(36.2)$ & \\
\hline & $\operatorname{UIIV}(\lambda)$ & 0.019 & $0.043(22.3)$ & \\
\hline & UIIV (o) & 1.09 & $0.34(31.2)$ & \\
\hline \multirow[t]{4}{*}{ Hybrid $_{\max }$ and Poisson } & $\mathrm{E}_{\max }$ & 11.8 & $0.26(2.17)$ & 2,752 \\
\hline & $\mathrm{ED}_{50}$ & 101 & $0.447(0.44)$ & \\
\hline & UIIV $\left(E_{\max }\right)$ & 0.322 & $0.02(6.41)$ & \\
\hline & $\operatorname{UIIV}\left(\mathrm{ED}_{50}\right)$ & 1.61 & $0.435(27)$ & \\
\hline \multirow[t]{4}{*}{ Linear } & Slope & 0.007 & $0.002(24.5)$ & 3,522 \\
\hline & Intercept & 7.16 & $0.376(5.25)$ & \\
\hline & UIIV (slope) & 0.008 & $0.001(12)$ & \\
\hline & UIIV (Intercept) & 1.94 & $0.216(11.2)$ & \\
\hline
\end{tabular}

$\mathrm{SE}$, standard error; RSE, relative standard error; AIC, Akaike information criterion; UIIV, unexplained interindividual variability; $E_{\text {max }}$, the maximum therapeutic effect; $\mathrm{ED}_{50}$, the dose associated with half-maximal therapeutic effect.

\section{Model validation}

The results obtained from 500 rounds of bootstrapping indicated a close match between the mean coefficients of the bootstrapped samples and those of the original model with little bias. All of the final model coefficients lay within the $95 \% \mathrm{Cl}$ of the mean coefficients estimated from bootstrapping, indicating stable and robust predictive performance of the final model. Table 4 summarizes the results of bootstrapping. Comparing the mean actual versus predicted oocyte count across the different studied gonadotropin doses resulted in nonsignificant differences, confirming the reliability of the model predictions (Figure 1).

The validation group $(n=139)$ demonstrated similar discrimination criteria compared to the index group (Table 5). For both groups, the developed model explained approximately $50 \%$ of the variability in the observed oocyte count (pseudo- $R^{2}: 0.48$ vs. 0.49 for the index and validation groups, respectively). The concluded model achieved statistical significance when analyzing both groups $(p<0.001$ for both groups). The C-statistic was reasonable for both models, con-

Table 4. Estimates from 500 bootstrapped samples of the original dataset

\begin{tabular}{lccc}
\hline Covariate & Mean coefficient & SE & $95 \% \mathrm{Cl}$ \\
\hline Age & -0.017 & 0.003 & -0.023 to -0.011 \\
AFC & 0.034 & 0.003 & 0.028 to 0.04 \\
FSH & -0.028 & 0.005 & -0.033 to 0.023 \\
Protocol type & \multicolumn{3}{|}{} \\
$\quad$ Long & \multicolumn{3}{c}{ Reference } \\
$\quad$ Short & 0.07 & 0.04 & -0.01 to 0.15 \\
$\quad$ Antagonist & 0.18 & 0.06 & 0.06 to -0.3 \\
Dose & 0.11 & 0.06 & -0.01 to 0.23
\end{tabular}

$\mathrm{SE}$, standard error; $\mathrm{Cl}$, confidence interval; $\mathrm{AFC}$, antral follicular count; $\mathrm{FSH}$, follicle-stimulating hormone.

Table 3. Regression analysis of oocyte retrieval versus candidate covariates

\begin{tabular}{|c|c|c|c|c|c|c|c|}
\hline \multirow{2}{*}{ Covariate } & \multicolumn{3}{|c|}{ Univariate regression } & \multicolumn{3}{|c|}{ Multivariate regression } & \multirow{2}{*}{ Included (yes/no) } \\
\hline & $r$ & Change in AIC & $p$-value & $r$ & $\mathrm{aOR}$ & $p$-value & \\
\hline Age & -0.028 & 85 & $<0.001$ & -0.019 & $0.98(0.976-0.987)$ & $<0.001$ & Yes \\
\hline AFC & 0.042 & 186 & $<0.001$ & 0.033 & $1.033(1.028-1.038)$ & $<0.001$ & Yes \\
\hline AMH & 0.053 & 23 & $<0.001$ & - & $0.15(0.99-1.04)$ & 0.21 & No \\
\hline Long & & & & erence & & & \\
\hline Short & 0.118 & \multirow{2}{*}{15} & 0.001 & 0.093 & $1.098(1.034-1.156)$ & 0.002 & \\
\hline Antagonist & 0.2 & & $<0.001$ & 0.208 & 1.231 (1.124-1.349) & $<0.001$ & \\
\hline Log (dose) & -0.221 & $N A^{a)}$ & $<0.001$ & 0.107 & $1.11(1.004-1.235)$ & 0.042 & \\
\hline
\end{tabular}

$r$, regression coefficient; AIC, Akaike information criterion; aOR, adjusted odds ratio; BMI, body mass index; AFC, antral follicular count; AMH, anti-Müllerian hormone; $\mathrm{FSH}$, follicle-stimulating hormone; NA, not applicable.

${ }^{a}$ The logarithm of the starting gonadotrophin dose is an essential component of the base structural model. 


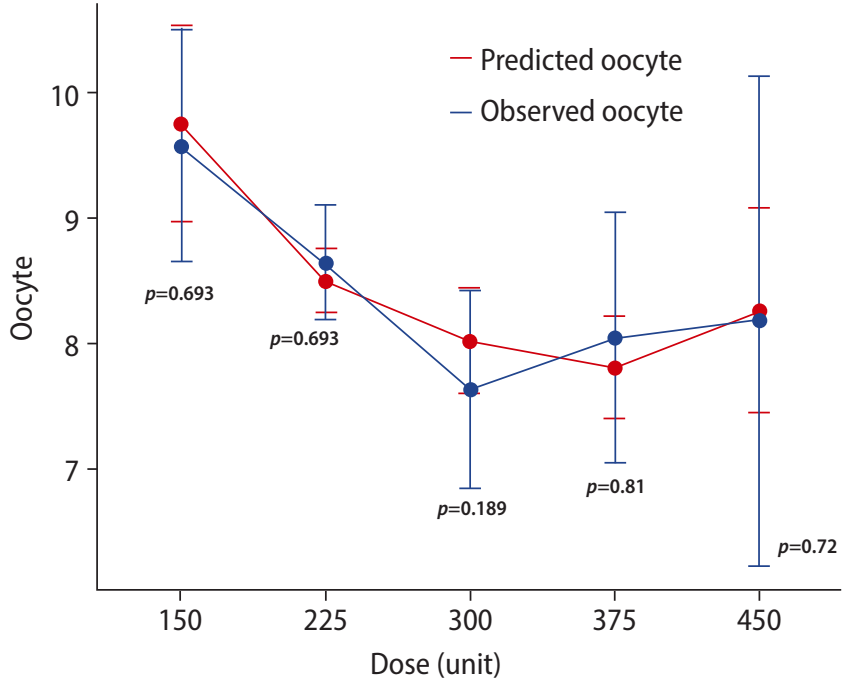

Figure 1. Comparison of the predicted versus observed oocyte yield for each gonadotropin dose level. Data are shown as mean \pm standard error of the mean. Error bars represent $95 \%$ confidence intervals. The Wilcoxon signed-rank test was applied to calculate the $p$-value for each pairwise comparison.

Table 5. Assessment of model performance in the validation group compared to the index group

\begin{tabular}{lcc}
\hline Parameter & Index group $(n=497)$ & Validation group $(n=139)$ \\
\hline RMSE $(\%)^{a)}$ & $3.04(34.88)$ & $3.88(43.11)$ \\
C-index $(\mathrm{SE})$ & $0.71(0.01)$ & $0.72(0.02)$ \\
Chi-square $(p$-value) & $319.28(<0.001)$ & $92(<0.001)$ \\
Pseudo- $R^{2 b)}$ & 0.48 & 0.49
\end{tabular}

$\mathrm{RMSE}$, relative mean squared error; $\mathrm{SE}$, standard error.

${ }^{\text {a) }}$ Estimated as exponents of log-linear mean squared error; ${ }^{\text {b) }}$ Calculated based on the Cragg-Uhler method.

firming the agreement between predicted and actual oocyte counts (C-statistic, 0.71 vs. 0.72 , respectively). The RMSE estimates for both models were also comparable. The estimated odds of oocyte yield from both models demonstrated a distinct similarity (Figure 2).

\section{Nomogram development}

Based on our validated final model, a point-based static nomogram was built to help individualize treatment decisions based on patient-specific characteristics (Figure 3). The prognostic weight of each independent variable is depicted by the length of the corresponding point scale. Gonadotropin dose and protocol type are the shortest scales; hence, they have a lower weight than the other patient-related factors. The nonlinear exposure-response relationship is well-captured by the point system scaling. The points attained from incremental increases of the gonadotropin dose non-linearly decrease as the dose increases; moreover, the total number of points required to increase the oocyte count is much higher in cases with a predicted retrieval of fewer than 8 oocytes than in those with a predicted retrieval of more than 15 oocytes (Figure 3). A dynamic individualization tool was constructed to aid electronic manipulation for more convenient institutional applications (Figure 4).

\section{Discussion}

The present study is the first to introduce a nomogram-based clinical tool that can be utilized to individualize both the protocol and the starting gonadotropin dose selection for IVF/ICSI candidates. The nomograms were built based on a robust validated model that predicted the number of retrieved oocytes from the stimulation cycles. In line with many previous studies $[8,18,19]$, the choice of retrieved oocytes as a target outcome to model was suggested in the current work to overcome confounding with different non-therapeutic factors encountered in models predicting IVF/ICSI success in terms of pregnancy or live birth rates. Moreover, the definition of response to COS in terms of absolute oocyte count enables a realistic representation of ovarian response as a continuous spectrum instead of "black and white" categories in binary logistic models for COS individualization $[11,12,20,21]$.

Our developed model identified women's age, basal FSH levels, AFC, stimulation protocol type, and gonadotropin dose as significant predictors of oocyte retrieval. The prognostic value of each of these identified predictors has been well established in previous reports $[8,11,12,22]$.

One of the main findings of the current model is that treatment-related factors, including the stimulation protocol and gonadotropin dose, may have little influence compared to the patient's intrinsic factors, including age and ORTs, when predicting oocyte retrieval. This suggests that the matter of optimal response to gonadotropin therapy is more related to the patient herself rather than the specific characteristics of the therapeutic approach. Similarly, Rustamov et al. [23] concluded that only $10 \%$ of the total observed variance of retrieved oocytes could be explained by treatment-related factors, while $53 \%$ could be attributed to patient-specific factors in their mixed-effects Poisson model.

Interestingly, the evaluation of the predictive performance of different ORTs demonstrated superior predictive performance of basal FSH levels compared to AMH levels. Similarly, Magnusson et al. [24] concluded that $\mathrm{AMH}$ levels did not improve the rates of targeted ovarian response when added to AFC. Nevertheless, the results of similar analyses should be interpreted with caution, especially when comparing our results, due to differences in sample sizes, the inclusion of confounding conditions (e.g., polycystic ovaries), and application of different stimulation protocols. For instance, Moon et al. [19] reported that age, basal FSH levels, AFC, and AMH levels were signifi- 


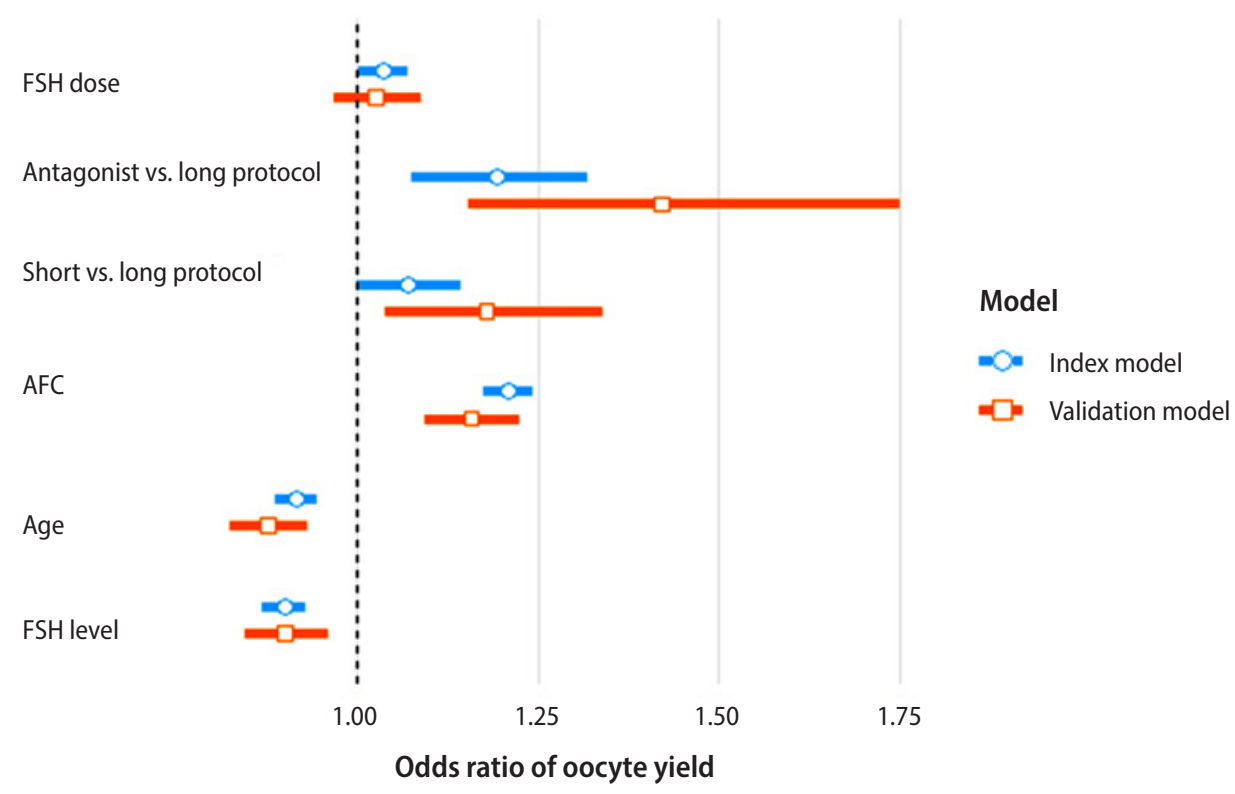

Figure 2. Forest plot comparing the odds ratios estimated from the index and validation models. Corresponding point estimates are depicted by blue circles (index group) or orange squares (validation model). Horizontal lines represent the corresponding $95 \%$ confidence intervals of the estimated odds ratios for each model. FSH, follicle-stimulating hormone; AFC, antral follicle count.

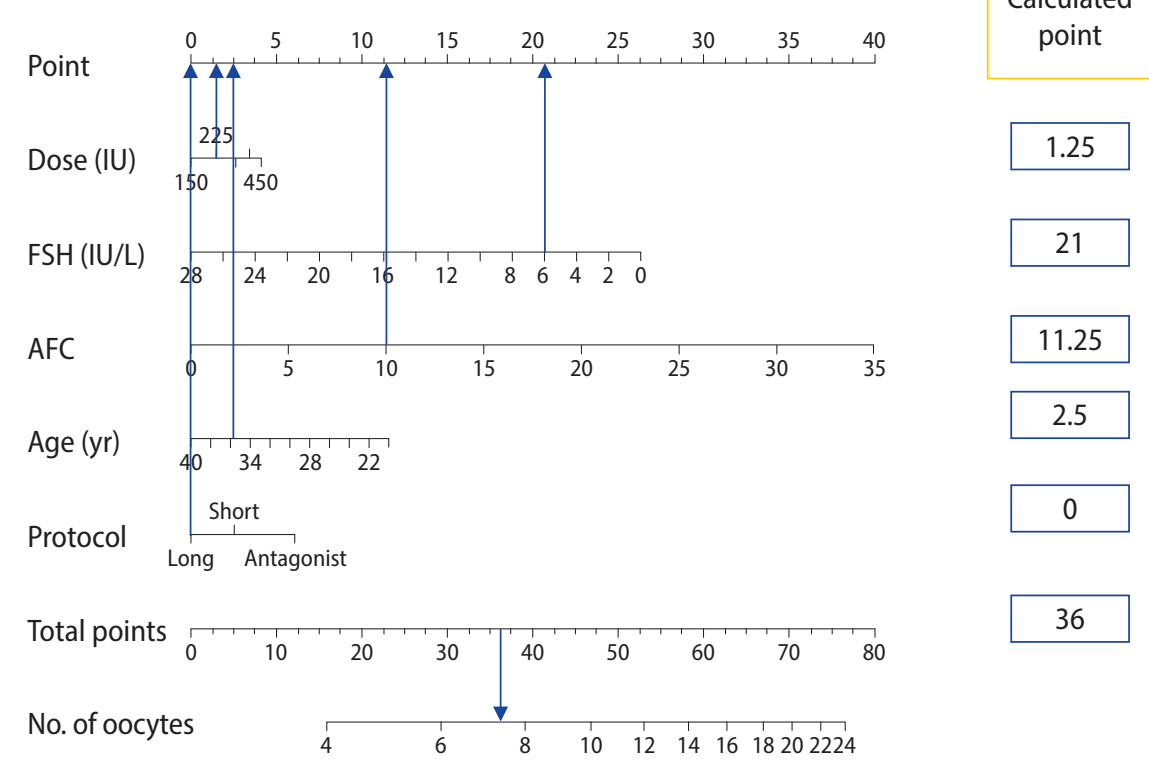

Figure 3. Static nomogram developed for individualizing controlled ovarian stimulation. In this example, a 36-year-old woman with an antral follicle count (AFC) of 10 and a basal follicle-stimulating hormone (FSH) level of $6 \mathrm{IU} / \mathrm{L}$ was evaluated for a starting empirical gonadotropin dose of $225 \mathrm{IU}$ as a part of long protocol. Based on our developed score, the woman was assigned a total of 36 points, corresponding to a predicted oocyte yield of fewer than 8 oocytes. To overcome the problem of poor response, a change in the stimulation protocol to an antagonist protocol only could be recommended (adding 6 points to the total score, thereby increasing the predicted oocyte count to 8 , corresponding to a good response). The instructions for the nomogram are as follows. (1) Based on the patient's specific data, extrapolate a vertical line from the specific values for each variable to the points on the axis in the upper panel. (2) For each variable, record the corresponding points in the corresponding boxes in the right panel. (3) Calculate the sum of assigned points and record the result in the box corresponding to the total points in the right panel. (4) Extrapolate a vertical line from the total points axis to the "no. of oocytes" axis, which gives an average prediction of oocyte count based on the selected treatment scenario. 


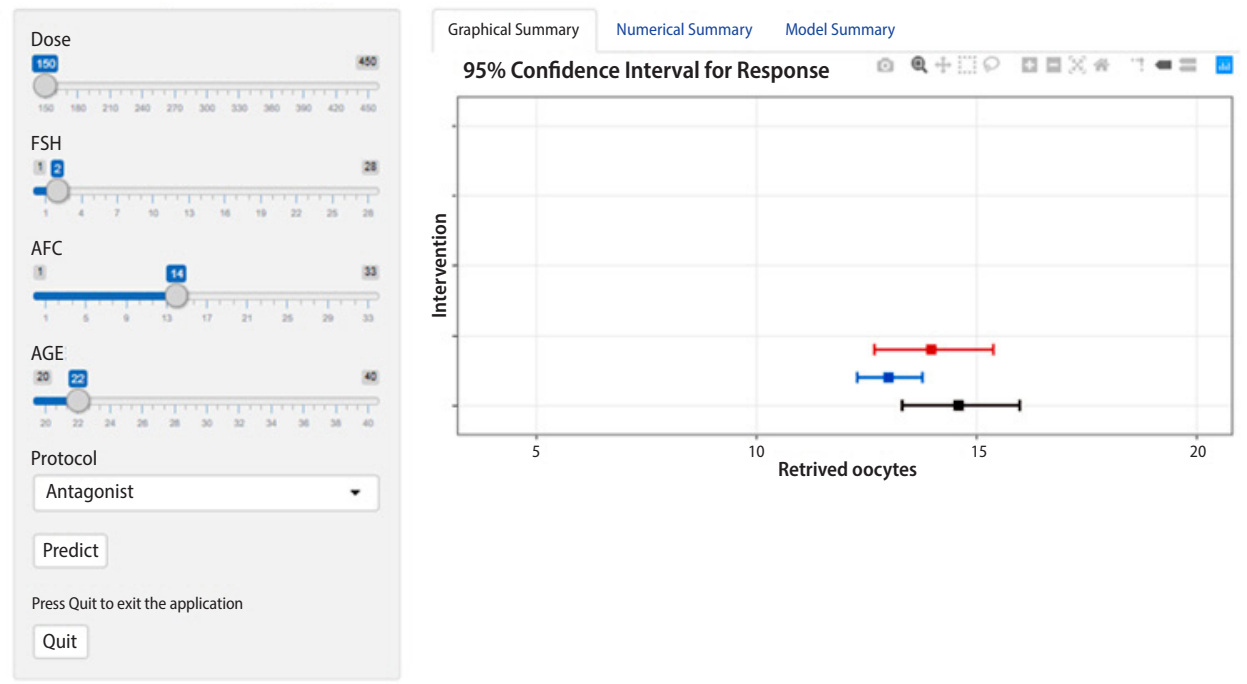

Figure 4. Application of the dynamic individualization tool in optimization for gonadotropin therapy. The black bar describes a predicted $95 \%$ confidence interval $(\mathrm{Cl})$ of the retrieved oocyte count based on a hypothetical selection of an antagonist protocol and a gonadotropin dose of $225 \mathrm{IU}$ daily for a 22-year-old woman with a basal follicle-stimulating hormone (FSH) level of $2 \mathrm{IU} / \mathrm{mL}$ and an antral follicle count (AFC) of 14 follicles. Due to the possibility of hyper-response, we note two possible modifications to the therapeutic regimen. First, the antagonist protocol can be changed to the short protocol (blue bar). Second, the dose can be reduced to $150 \mathrm{IU}$ (red bar). Both modifications seem to be safe (the mean predicted oocyte count is between 8 and 14 oocytes); however, changing the protocol seems to be safer, as there would be no risk of hyper-response (the $95 \% \mathrm{Cl}$ of oocyte yield is less than 15). By clicking on the numerical summary tab on the upper right panel, a detailed summary of the three scenarios is provided. The developed tool is freely available at https://individualization.shinyapps.io/ dynnomapp/ for institutional use [25].

cant predictors of the oocyte count; however, in their final model, they excluded FSH due to its relatively weak predictive performance. This low weight of basal FSH may be related to their relatively small sample size $(n=141)$, and the high inter-subject variation that was encountered with FSH levels in their work (range, $2.9-26.6 \mathrm{mlU} / \mathrm{mL}$; coefficient of variation, 45\%) [19].

In particular, the impact of gonadotropin dose on oocytes remains debatable. It was suggested that a positive relationship may exist between gonadotropin doses from $50 \mathrm{IU}$ to $225 \mathrm{IU}$ and oocyte yield, whereas doses more than $225 \mathrm{IU}$ failed to demonstrate an extra benefit, indicating a possible nonlinear relationship [26]. Possible explanations for this phenomenon include limited capacity of the follicular pool, large variability in the AFC, and/or sensitivity to exogenous gonadotropin stimulation [27]. Unfortunately, the characterization of the nonlinear dose-response relationship has largely been overlooked in previous similar reports. It is therefore not surprising that our results identified the linear models as the worst ones, highlighting a major criticism of the currently published linear regression models for gonadotropin dose individualization [28-30]. In the current study, two possible models were proposed to capture these nonlinear patterns: hybrid $\mathrm{E}_{\max }$ and modified Poisson models. In pharmacodynamic terms, the $\mathrm{E}_{\max }$ model assumes that a drug has a maximum effect at a certain dose, while beyond this dose, no addi- tional response will be obtained. The $\mathrm{E}_{\max }$ model is parameterized with $E_{\max }$ a metric for the average maximum response with escalating FSH doses, and $\mathrm{ED}_{50}$, a metric that corresponds to the dose that yields half of the maximum response [31]. The idea behind our proposed hybrid $\mathrm{E}_{\max }$ model is that the traditional version of $\mathrm{E}_{\max }$ predicts continuous responses, which is not suitable for modeling oocytes as discrete outcomes [16], while the Poisson model correctly predicts oocyte count as a discrete variable, but assumes a linear relationship between FSH dose and oocyte count, which is not true [19]. In the proposed hybrid model, we replaced the traditional exponential linear function with the exponential nonlinear $E_{\max }$ function to account for the relatively flat dose-response relationship attained at higher FSH doses, while enabling oocytes to be predicted as discrete counts. In contrast, the proposed modified Poisson model introduced the FSH dose covariate in log terms instead of the linear scale implemented in conventional Poisson models to account for the nonlinear FSH dose-oocyte count relationship. Comparing both models, the modified Poisson model demonstrated the best fit with the highest precision and showed a significant effect of gonadotropin dose on oocytes $(p=0.042)$.

In contrast to our results, a recent meta-analysis concluded that escalating FSH doses had similar efficacy and safety profiles in both poor and hyper-responders. However, the authors reported that the 
included studies might have suffered from limited sample sizes and heterogeneous comparisons of different doses of FSH [32]. Borges et al. [29] demonstrated that escalating doses of FSH were not associated with an increased oocyte count. However, they reported lower FSH requirements per oocyte retrieved at a younger age ( $\leq 35$ years), suggesting that the impact of gonadotropin dose on oocytes may be masked by intrinsic poor ovarian function, primarily due to aging.

Regarding the protocol type, our findings indicate that milder protocols were associated with higher oocyte yield than the conventional long stimulation protocol. This finding is supported by Pinto et al. [20], who found that antagonist protocols were associated with a significantly higher mean number of retrieved oocytes than long protocols. In contrast, three different meta-analyses reported statistically significant lower oocyte yields with antagonist protocols than with agonist (long) protocols [33-35]. Of note, Lambalk et al. [35] demonstrated a non-significant difference among protocol types in poor responders, supporting our theory that the intrinsic response to gonadotropins is basically related to the characteristics of the patient herself. To our knowledge, no study has adjusted for the effect of the higher gonadotropin doses involved in the long protocol. It should be noted that the longer duration of stimulation, with the subsequent higher total gonadotropin dose per cycle in the long protocol, may potentially confound this relationship [14]. Whether a higher oocyte count is related to the specific type of stimulation or the higher gonadotropin dose in the long protocol remains questionable.

To translate models into practice, nomograms provide rigorous tools for model visualization, helping clinicians to optimize treatment decisions on an individual basis [36]. The nomogram-based model for oocyte prediction previously introduced by La Marca et al. [8] was limited by the analysis of a homogeneous group of predicted normal responders only, with a single stimulation protocol (long protocol only). In contrast to our model findings, they demonstrated enhanced predictive performance of their multivariate linear model, which reached up to $50 \%$ correct FSH dose prediction in predicted normal responders aged less than 35 years. Moreover, the external validation of the nomograms developed by La Marca et al. [8] resulted in acceptable performance in individualizing FSH doses [7,37]. This suggests that the application of linear models in predicting a suitable gonadotropin dose may be appropriate when analyzing a more homogeneous population of predicted normal responders. Despite comparable predictive performance, our results should be interpreted with great caution when compared to the findings of La Marca et al. [8] due to the implementation of a different dose-response model and the analysis of a more heterogeneous group that included different patterns of response other than predicted normal response. More recently, Moon et al. [19] presented an appropriate oocyte model in terms of Poisson regression for counts; however, their nomogram was criticized for failure to interpret the contributions of both protocol type and gonadotropin doses to oocyte retrieval due to their relatively small sample size.

Taken together, the current study exhibits several strengths. First, the fitted model adequately describes the complex nonlinear relationship between gonadotropin doses and oocyte retrieval. Second, the developed model dealt with the large heterogeneity in the definitions of ovarian response by modeling the response to $\operatorname{COS}$ as the absolute oocyte count. Third, the developed nomogram is user-friendly and does not require the user to apply any sophisticated mathematical or statistical techniques, as were used in the process of model building. Fourth, our nomogram is the first to introduce a method for integrated gonadotropin therapy personalization, enabling both the protocol and subsequent gonadotropin dose to be tailored. Nevertheless, the current work has several limitations. Since external validation was not possible during the development phase, the generalizability of the proposed nomogram remains unknown. The exclusion of women with explained causes of female infertility further limits the generalizability of the developed nomogram. The model used for oocyte retrieval did not account for intra-cycle variability due to its dependence on baseline clinical and demographic characteristics only. Additionally, the literature has presented many factors that may have an influence on oocyte prediction, including estradiol levels, FSHR gene polymorphisms, and smoking status, which were unavailable for the included women during the data collection phase. Since the study population included only naïve IVF/ ICSI candidates, the role of previous stimulation cycles in predicting oocyte count could not be investigated in the current work. The retrospective nature of the study may pose a threat of information bias. The retrospective design allowed data collection from a relatively large population for optimal model building. The exact predictive performance of the total gonadotropin doses administered during the entire cycle could not be investigated due to a lack of monitoring of patient adherence to the prescribed gonadotropin therapy, which might have resulted in mismatching between the recorded doses and the actual administered doses. Similarly, this point could be raised to criticize previous studies [19,38], which assessed the effect of total gonadotropin doses on ovarian response without reporting how patient adherence was monitored, introducing a risk of potential information bias [39]. Future studies should apply intensive monitoring schedules for different ORTs during IVF/ICSI cycles for further validation of our nomograms with more detailed data collection to address all possible sources of oocyte yield variability. 


\section{Conflict of interest}

No potential conflict of interest relevant to this article was reported.

\section{ORCID}

$\begin{array}{ll}\text { Abdel Hameed I M Ebid } & \text { https://orcid.org/0000-0002-9072-0815 } \\ \text { Sara M Abdel Motaleb } & \text { https://orcid.org/0000-0003-0836-3706 } \\ \text { Mahmoud I Mostafa } & \text { https://orcid.org/0000-0002-3247-5364 } \\ \text { Mahmoud M A Soliman } & \text { https://orcid.org/0000-0002-3268-7412 }\end{array}$

\section{Author contributions}

Conceptualization: AHIME, Formal analysis: MIM. Methodology: SMAM, Writing-original draft: MIM. Writing-review \& editing: MMAS.

\section{References}

1. Farquhar C, Marjoribanks J. Assisted reproductive technology: an overview of Cochrane Reviews. Cochrane Database Syst Rev 2018:8:CD010537.

2. Lazer T, Dar S, Shlush E, Al Kudmani BS, Quach K, Sojecki A, et al. Comparison of IVF outcomes between minimal stimulation and high-dose stimulation for patients with poor ovarian reserve. Int J Reprod Med 2014;2014:581451.

3. Toftager M, Bogstad J, Bryndorf T, Lossl K, Roskaer J, Holland T, et al. Risk of severe ovarian hyperstimulation syndrome in $\mathrm{GnRH}$ antagonist versus $\mathrm{GnRH}$ agonist protocol: RCT including 1050 first IVF/ICSI cycles. Hum Reprod 2016;31:1253-64.

4. Siristatidis C, Salamalekis G, Dafopoulos K, Basios G, Vogiatzi P, Papantoniou N. Mild versus conventional ovarian stimulation for poor responders undergoing IVF/ICSI. In Vivo 2017;31:231-7.

5. Arce JC, Andersen AN, Fernandez-Sanchez M, Visnova H, Bosch E, Garcia-Velasco JA, et al. Ovarian response to recombinant human follicle-stimulating hormone: a randomized, antimüllerian hormone-stratified, dose-response trial in women undergoing in vitro fertilization/intracytoplasmic sperm injection. Fertil Steril 2014;102:1633-40.

6. Nyboe Andersen A, Nelson SM, Fauser BC, Garcia-Velasco JA, Klein BM, Arce JC, et al. Individualized versus conventional ovarian stimulation for in vitro fertilization: a multicenter, randomized, controlled, assessor-blinded, phase 3 noninferiority trial. Fertil Steril 2017;107:387-96.

7. Allegra A, Marino A, Volpes A, Coffaro F, Scaglione P, Gullo S, et al. A randomized controlled trial investigating the use of a predictive nomogram for the selection of the FSH starting dose in IVF/ICSI cycles. Reprod Biomed Online 2017;34:429-38.

8. La Marca A, Grisendi V, Giulini S, Argento C, Tirelli A, Dondi G, et al. Individualization of the FSH starting dose in IVF/ICSI cycles using the antral follicle count. J Ovarian Res 2013;6:11.

9. Oliveira JB, Baruffi RL, Petersen CG, Mauri AL, Nascimento AM,

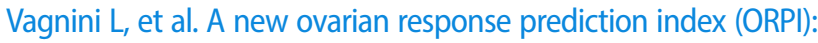
implications for individualised controlled ovarian stimulation. Reprod Biol Endocrinol 2012;10:94.

10. La Marca A, Sunkara SK. Individualization of controlled ovarian stimulation in IVF using ovarian reserve markers: from theory to practice. Hum Reprod Update 2014;20:124-40.

11. Oehninger S, Nelson SM, Verweij P, Stegmann BJ. Predictive factors for ovarian response in a corifollitropin alfa/GnRH antagonist protocol for controlled ovarian stimulation in IVF/ICSI cycles. Reprod Biol Endocrinol 2015;13:117.

12. Vuong TN, Vo MT, Ho MH. Predictive value of $A M H, F S H$ and AFC for determining ovarian response in Vietnamese women undergoing assisted reproductive technologies: a prospective study. J Fertil In Vitro IVF Worldw Reprod Med Genet Stem Cell Biol 2015; 3:151.

13. Altman DG, Royston P. The cost of dichotomising continuous variables. BMJ 2006;332:1080.

14. Ou J, Xing W, Li Y, Xu Y, Zhou C. Short versus long gonadotropin-releasing hormone analogue suppression protocols in IVF/ ICSI cycles in patients of various age ranges. PLoS One 2015;10: e0133887.

15. Islam Y, Aboulghar MM, AlEbrashy AE, Abdel-Aziz O. The value of different ovarian reserve tests in the prediction of ovarian response in patients with unexplained infertility. Middle East Fertil Soc J 2016;21:69-74.

16. Felmlee MA, Morris ME, Mager DE. Mechanism-based pharmacodynamic modeling. Methods Mol Biol 2012;929:583-600.

17. Plan EL. Modeling and simulation of count data. CPT Pharmacometrics Syst Pharmacol 2014;3:e129.

18. Al-Azemi M, Killick SR, Duffy S, Pye C, Refaat B, Hill N, et al. Multi-marker assessment of ovarian reserve predicts oocyte yield after ovulation induction. Hum Reprod 2011;26:414-22.

19. Moon KY, Kim H, Lee JY, Lee JR, Jee BC, Suh CS, et al. Nomogram to predict the number of oocytes retrieved in controlled ovarian stimulation. Clin Exp Reprod Med 2016;43:112-8.

20. Pinto F, Oliveira C, Cardoso MF, Teixeira-da-Silva J, Silva J, Sousa M, et al. Impact of GnRH ovarian stimulation protocols on intracytoplasmic sperm injection outcomes. Reprod Biol Endocrinol 2009; 7:5.

21. Lukaszuk K, Kunicki M, Liss J, Lukaszuk M, Jakiel G. Use of ovarian reserve parameters for predicting live births in women undergoing in vitro fertilization. Eur J Obstet Gynecol Reprod Biol 2013; 
168:173-7.

22. Tsakos E, Tolikas A, Daniilidis A, Asimakopoulos B. Predictive value of anti-müllerian hormone, follicle-stimulating hormone and antral follicle count on the outcome of ovarian stimulation in women following GnRH-antagonist protocol for IVF/ET. Arch Gynecol Obstet 2014;290:1249-53.

23. Rustamov O, Wilkinson J, La Marca A, Fitzgerald C, Roberts SA. How much variation in oocyte yield after controlled ovarian stimulation can be explained? A multilevel modelling study. Hum Reprod Open 2017;2017:hox018.

24. Magnusson A, Nilsson L, Olerod G, Thurin-Kjellberg A, Bergh C. The addition of anti-Müllerian hormone in an algorithm for individualized hormone dosage did not improve the prediction of ovarian response-a randomized, controlled trial. Hum Reprod 2017;32:811-9.

25. Gonadotrophin therapy individualization tool [Internet]. 2021 [cited 2021 May 1]. Available at: https://individualization.shinyapps. io/dynnomapp/.

26. Broekmans FJ. Individualization of FSH doses in assisted reproduction: facts and fiction. Front Endocrinol (Lausanne) 2019;10:181.

27. Drakopoulos P, Santos-Ribeiro S, Bosch E, Garcia-Velasco J, Blockeel C, Romito A, et al. The effect of dose adjustments in a subsequent cycle of women with suboptimal response following conventional ovarian stimulation. Front Endocrinol (Lausanne) 2018;9:361.

28. Hashish NM, Shaeer EK. Choosing the optimal dose of human menopausal gonadotropins for ovarian stimulation in ICSI cycles. Middle East Fertil Soc J 2014;19:124-8.

29. Borges E Jr, Zanetti BF, Setti AS, Braga DP, Figueira RC, laconelli A Jr. FSH dose to stimulate different patient' ages: when less is more. JBRA Assist Reprod 2017;21:336-42.

30. Zhu M, Wang S, Yi S, Huang X, Meng J, Chen L, et al. A predictive formula for selecting individual FSH starting dose based on ovarian reserve markers in IVF/ICSI cycles. Arch Gynecol Obstet 2019;300:4416.
31. Salahudeen MS, Nishtala PS. An overview of pharmacodynamic modelling, ligand-binding approach and its application in clinical practice. Saudi Pharm J 2017;25:165-75.

32. Lensen SF, Wilkinson J, Leijdekkers JA, La Marca A, Mol BW, Marjoribanks J, et al. Individualised gonadotropin dose selection using markers of ovarian reserve for women undergoing in vitro fertilisation plus intracytoplasmic sperm injection (IVF/ICSI). Cochrane Database Syst Rev 2018;2:CD012693.

33. Xiao JS, Su CM, Zeng XT. Comparisons of GnRH antagonist versus $\mathrm{GnRH}$ agonist protocol in supposed normal ovarian responders undergoing IVF: a systematic review and meta-analysis. PLoS One 2014;9:e106854.

34. Al-Inany HG, Youssef MA, Ayeleke RO, Brown J, Lam WS, Broekmans FJ. Gonadotrophin-releasing hormone antagonists for assisted reproductive technology. Cochrane Database Syst Rev 2016:4:CD001750.

35. Lambalk CB, Banga FR, Huirne JA, Toftager M, Pinborg A, Homburg $\mathrm{R}$, et al. GnRH antagonist versus long agonist protocols in IVF: a systematic review and meta-analysis accounting for patient type. Hum Reprod Update 2017;23:560-79.

36. Van Belle V, Van Calster B. Visualizing risk prediction models. PLoS One 2015;10:e0132614.

37. Papaleo E, Zaffagnini S, Munaretto M, Vanni VS, Rebonato G, Grisendi $\mathrm{V}$, et al. Clinical application of a nomogram based on age, serum FSH and $\mathrm{AMH}$ to select the FSH starting dose in IVF/ICSI cycles: a retrospective two-centres study. Eur J Obstet Gynecol Reprod Biol 2016;207:94-9.

38. Zhou J, Wang B, Hu Y, Sun H. Association between the number of oocytes retrieved and cumulative live birth rate in women aged 35-40 years undergoing long GnRH agonist IVF/ICSI cycles. Arch Gynecol Obstet 2017;296:1005-12.

39. Farmer KC. Methods for measuring and monitoring medication regimen adherence in clinical trials and clinical practice. Clin Ther 1999:21:1074-90. 